\title{
Characterization of the ballistic limit curve for metallic Whipple shield
}

\author{
M. Lee ${ }^{\text {a }}$
}

\author{
Sejong University, Mechanical Engineering, 98 Kunja-Dong , Seoul 143-747, Korea
}

\begin{abstract}
It has been known that space debris or meteoroid impact damage can have significant effects on spacecraft. Experimental test has been conducted up to $7 \mathrm{~km} / \mathrm{s}$, and numerical simulations are performed at higher velocities. Studies on the hypervelocity impact onto single plate, double spaced plates (Whipple shield), and multiple plates (MS shield) have been performed and ballistic limit curves (BLCs) are proposed. Last 15 years SPH (Smoothed Particle Hydrodynamics) has been applied to the hypervelocity impact problems because of cost of test and numerical efficiency especially in the hypervelocity impact regime. Although most of the simulations captured the debris shape well, somehow they do not seem to match well with the empirical ballistic limit curves. We have recently developed a new axisymmetric SPH hydrocode. In order to assess the confidence that should be placed in such simulations we simulated the hypervelocity impacts on aluminum Whipple shields and compared with the empirical BLCs. The SPH simulations indicated an improved accuracy compared with the previously published SPH simulation results. Other effort we put was using different types of equation of state, however no further improvement was achieved.
\end{abstract}

\section{Introduction}

Understanding and controlling large plastic deformation problems such as hypervelocity impact/penetration (roughly $\mathrm{V}>2 \mathrm{~km} / \mathrm{s}$ ) events are of great importance for protection of space vehicles and satellite system as well. This is becasue the external walls are exposed to impacts from meteoroid or space debris [1]. In these areas, experimental test has been conducted up to $7 \mathrm{~km} / \mathrm{s}$, and numerical simulations are performed for higher velocities. For protection, one uses dual-plate shields [2] or multishock shields [3]. Details of the characteristics of debris clouds produced by hypervelocity impact of aluminum spheres with thin aluminum plates were described using flash radiographs [4]. Some studies were conducted for Whipple shield and nice empirical equations for ballistic limit are available in the literature $[5,6]$.

Numerical efforts were made to reproduce this ballistic limit curve (BLC). However, the predictions show some discrepancy. Palmieri et al [7] tried to use different equations of state (Shock, Tilloston, and Sesame 3715). This is because the Sesame tables cover a very wide range of material strain rates, including liquid and vapor phase transitions and the Tilloston can also be applied much higher pressure ranges. However they do not seem to do better job compared to the generic linear Shock EOS. A numerical study was also conducted to reproduce the BLC [8] using a Mie-Grunisen form with phase changes from solid to liquid and vapor, plus an ionization model. Again no better matching was indicated.

In this paper, we simulated the metallic Whipple shield with our in-house SPH hydrocode. A significant amount of time was spent to eliminate any bug in the axi-symmetric code. In fact, some errors or type-o are available in the references. A relatively better agreement has been obtained even though a generic linear Shock EOS is used for the materials. Most of all, extreme care should be taken in determining the threshold penetration (ballistic limit, spall limit) in simulations since at this threshold the velocity of the back plate becomes much small.

a e-mail: mlee@sejong.ac.kr

\section{Axi-symmetric SPH}

\subsection{Introduction}

Lucy [9], Gingold and Monaghan [10] first introduced the Smooth Particle Hydrodynamics (SPH) in space science areas. SPH scheme was adopted and applied to the computations of large deformation problems $[11,12]$ Libersky and Petschek 1990, Johnson et al. 1993). Johnson et al. [13], and Hayhurst et al. [14] implemented relatively simple algorithms for axisymmetric geometry. These are much similar in that they consider each particle as torus ring geometry. The cylindrical SPH code we developed recently is much simple to migrate from a Cartesian coordinate version. A brief discussion of the mathematical description is provided below.

\subsection{Mathematical formulation}

The internal forces in solids with material strength are governed by the following conservation equations. in Cartesian coordinates;

$$
\begin{aligned}
\frac{D \rho}{D t} & =-\rho\left(\frac{\partial u^{x}}{\partial x}+\frac{\partial u^{y}}{\partial y}\right) \\
\frac{D u^{x}}{D t} & =\frac{1}{\rho}\left(\frac{\partial \sigma^{x x}}{\partial x}+\frac{\partial \sigma^{x y}}{\partial y}\right) \\
\frac{D u^{y}}{D t} & =\frac{1}{\rho}\left(\frac{\partial \sigma^{x y}}{\partial x}+\frac{\partial \sigma^{y y}}{\partial y}\right)
\end{aligned}
$$

in cylindrical coordinates;

$$
\begin{aligned}
\frac{D \rho}{D t} & =-\rho\left(\frac{\partial u^{r}}{\partial r}+\frac{\partial u^{r}}{\partial r}+\frac{u^{r}}{r}\right) \\
\frac{D u^{r}}{D t} & =\frac{1}{\rho}\left(\frac{\partial \sigma^{r r}}{\partial r}+\frac{\partial \sigma^{r z}}{\partial z}+\frac{\sigma^{r r}-\sigma^{\theta \theta}}{r}\right) \\
\frac{D u^{z}}{D t} & =\frac{1}{\rho}\left(\frac{\partial \sigma^{r z}}{\partial r}+\frac{\partial \sigma^{z z}}{\partial z}+\frac{\sigma^{r z}}{r}\right)
\end{aligned}
$$

This is an Open Access article distributed under the terms of the Creative Commons Attribution License 2.0, which permits unrestricted use, distribution, and reproduction in any medium, provided the original work is properly cited. 

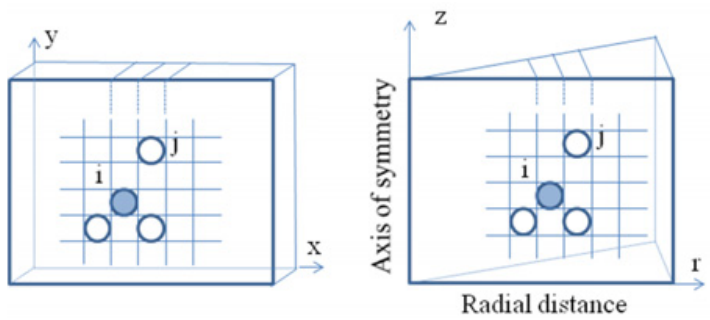

Fig. 1. Particle representation in two-dimensional plain strain and axisymmetric geometries.

where $\sigma$ is the stress tensor and $u$ is the velocity. $x, y$ are the Cartesian coordinates and $r, z$ are the cylindrical coordinates in radial- and axial direction. These equations are to be switched depending on the geometry chosen. A commonly used expression, in the Cartesian coordinates, which is converted to a particle approximation via a kernel function, $W$, is

$$
\begin{aligned}
& \frac{D \rho_{i}}{D t}=\sum_{j=1}^{N} m_{j}\left[\left(u_{i}^{x}-u_{j}^{x}\right) \frac{\partial W_{i j}}{\partial x_{\mathrm{i}}}+\left(u_{i}^{y}-u_{j}^{y}\right) \frac{\partial W_{i j}}{\partial y_{\mathrm{i}}}\right] \\
& \frac{D u_{i}^{x}}{D t}=-\sum_{j=1}^{N} m_{j}\left[\left(\frac{\sigma_{i}^{x x}}{\rho_{i}^{2}}+\frac{\sigma_{j}^{x x}}{\rho_{j}^{2}}\right) \frac{\partial W_{i j}}{\partial x_{\mathrm{i}}}+\left(\frac{\sigma_{i}^{x y}}{\rho_{i}^{2}}+\frac{\sigma_{j}^{x y}}{\rho_{j}^{2}}\right) \frac{\partial W_{i j}}{\partial y_{\mathrm{i}}}\right] \\
& \frac{D u_{i}^{y}}{D t}=-\sum_{j=1}^{N} m_{j}\left[\left(\frac{\sigma_{i}^{x y}}{\rho_{i}^{2}}+\frac{\sigma_{j}^{x y}}{\rho_{j}^{2}}\right) \frac{\partial W_{i j}}{\partial x_{\mathrm{i}}}+\left(\frac{\sigma_{i}^{y y}}{\rho_{i}^{2}}+\frac{\sigma_{j}^{y y}}{\rho_{j}^{2}}\right) \frac{\partial W_{i j}}{\partial y_{\mathrm{i}}}\right]
\end{aligned}
$$

where $N$ is the number of pairs which are identified to exist within the smoothing length (influence zone), $h$. Here $i$ and $j$ represent the center particle and the neighbouring particles. The smoothing function value is obtained by the summation of the neighbouring particles where the influence is varying with the distance. The most widely used cubic B-spline smoothing function is expressed as,

$$
W(R, h)=c_{d} \begin{cases}2 / 3-R^{2}+1 / 2 R^{3} & 0 \leq R<1 \\ 1 / 6(2-R)^{3} & 1 \leq R<2 \\ 0 & 2 \leq R\end{cases}
$$

where $R$ is the relative distance between two particles with respect to $h$, that is $R=\sqrt{\left(\left(x_{i}-x_{j}\right)^{2}+\left(y_{i}-y_{j}\right)^{2}\right)} / h$. Here $c_{d}$ is a constant equal to,

$$
c_{d}= \begin{cases}1 / h & \text { one }- \text { dimension } \\ 15 /\left(7 \pi h^{2}\right) & \text { two }- \text { dimension } \\ 3 /\left(2 \pi h^{3}\right) & \text { three }- \text { dimension }\end{cases}
$$

This constant is determined such that the unit of $W$ becomes the inverse of volume in each dimension in Cartesian coordinates. This can lead to inconsistency when used in the cylindrical geometry.

A major difference is caused by the representation of mass of a cylindrical particle. As it is torus ring geometry (refer to figure 1), its volume (mass) is in fact a threedimensional one,

$$
d V=(2 \pi r) d r d z, d m=(2 \pi r) \rho d r d z
$$

In the previous method $[13,14]$, the axisymmetric effect comes from dividing mass by $(2 \pi r)$ to get the equivalent masses for plain strain whenever $m$ (mass) appears in the particle approximations, while keeping the same smoothing function for both the Cartesian and axisymmetric geometries

Now we like to propose a simple method for migration of a Cartesian coordinate code into an axisymmetric version. We go back to the fundamental principle of SPH that can be obtained in the form of the discretized particle approximation.

in Cartesian coordinates;

$$
\begin{aligned}
f(x) & =\int_{\Omega} f\left(x^{\prime}\right) W\left(x-x^{\prime}, h\right) d x^{\prime} \\
& =\int_{\Omega} f\left(x^{\prime}\right) W\left(x-x^{\prime}, h\right) d x d y \\
& \approx \sum_{j=1}^{N} f\left(x_{j}\right) W\left(x-x_{j}, h\right) \Delta V_{j} \\
& =\sum_{j=1}^{N} \frac{m_{j}}{\rho_{j}} f\left(x_{j}\right) W\left(x-x_{j}, h\right) \\
d V_{j} & =(1)(\Delta x)(\Delta y)
\end{aligned}
$$

in cylindrical coordinates;

$$
\begin{aligned}
f(x) & =\int_{\Omega} f\left(x^{\prime}\right) W\left(x-x^{\prime}, h\right) d x^{\prime} \\
& =\int_{\Omega} f\left(x^{\prime}\right) W\left(x-x^{\prime}, h\right) r d \theta d r d z \\
& \approx \sum_{j=1}^{N} f\left(x_{j}\right) W\left(x-x_{j}, h\right) \Delta V_{j} \\
& =\sum_{j=1}^{N} \frac{m_{j}}{\rho_{j}} f\left(x_{j}\right) W\left(x-x_{j}, h\right) \\
d V_{j} & =\left(2 \pi r_{j}\right)(\Delta x)(\Delta y)
\end{aligned}
$$

As demonstrated in Eq. (7), in Cartesian coordinates the unit of $W$ should be the inverse of two-dimensional volume and Eq. (5) satisfies this requirement. In the cylindrical $\mathrm{SPH}$, however, if we use the same coefficient in smoothing function mismatch occurs in this approximation since $d V_{j}\left(m_{j}\right)$ is in fact a three-dimensional volume (mass). A remedy can be included directly into the calculation of the smoothing function as below.

$$
W_{i j}^{\text {axisymmetric }}=W_{i j} /\left(2 \pi r_{i}\right)
$$

Now the unit consistency is recovered and at the same time it is guaranteed that the neighbouring particle $(j)$ at large radial location has bigger volume (mass) by the factor of $r_{j} / r_{i}$. The same is true to the particle approximation for the spatial derivative of the function values. In conclusion, this method allows us an easy incorporation of the cylindrical SPH scheme since it is not necessary to change any other equations used in the Cartesian SPH formulation. 


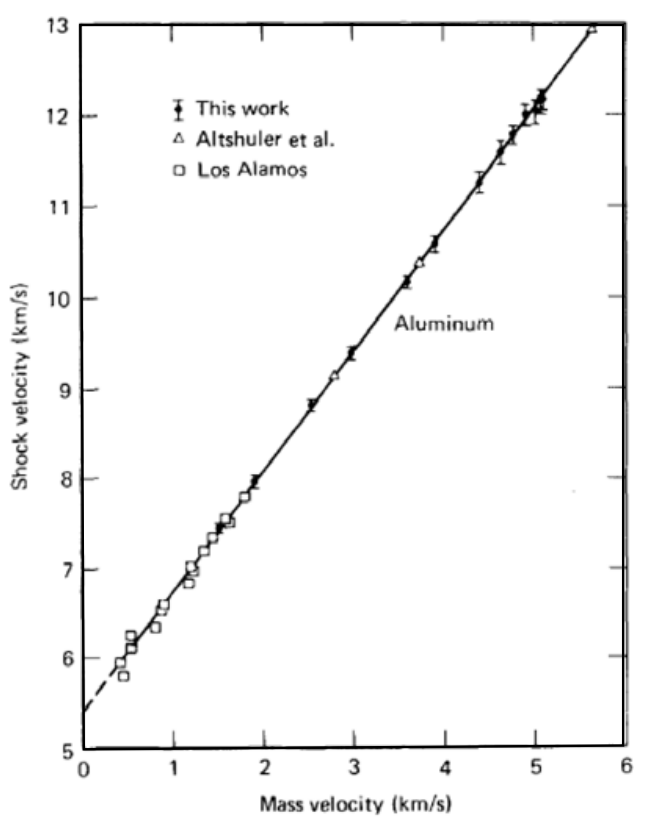

Fig. 2. Shock velocity vs. mass velocity for $\mathrm{Al}$ [15].

\section{Numerical modelling}

A series of SPH simulations has been conducted for impact of an aluminum sphere onto a aluminum spcaed double plates at normal incidencce. All was performed in two-dimensional axisymmetric domains. The impact velocity was parametrically varied in a series of numerical simulations from $2 \mathrm{~km} / \mathrm{s}$ to $14 \mathrm{~km} / \mathrm{s}$. The bumper thickness is $1.6 \mathrm{~mm}$, spacing $120 \mathrm{~mm}$ and the back wall $3.2 \mathrm{~mm}$. The radial extent of the bumper is 10 times of the thickness $(16 \mathrm{~mm})$. That of the backwall is $50 \mathrm{~mm}$. This target configuration was kept fixed throughout the simulations. To determine the threshold, each time the diameter of projectile sphere is increased by $0.01 \mathrm{~mm}$, such that the resolution of the critical diameter is $\pm 0.005 \mathrm{~mm}$.

The critical sphere diameter are found to be between $3 \mathrm{~mm}$ and $9 \mathrm{~mm}$. We put at least 10 particles across the bumper thickness, giving a bumper particle size of $0.16 \mathrm{~mm}$. We tried to maintain a consistent mesh resolution system in all cases. For the backwall, then it is packed with 20 particles across the thickness. The ratio of bumper particle size to backwall particle size was less than one, roughly $0.7 \sim 1$, but is attempted to be of comparable sizes. The radial extent of the backwall plate is much larger than the bumper plate. A minimum of 10,000 particles and a maximum of 20,000 particles were used. The constitutive response for $6061 \mathrm{~T} 6$ Aluminum was represented by the Johnson-Cook model. The shock equation of state (EOS) is used in this study.

$$
u_{s}=c+S u_{p}
$$

As shown in Fig. 2, it is not to use two linear curves to represent shock velocities as a function of particle velocity even up to $13 \mathrm{~km} / \mathrm{s}$ [15]. Actually melting is observed in this range. We tested with two curves, resulting in no significant difference in the simulations. The values used
Table 1. Material properties for the Aluminum.

\begin{tabular}{|l|c|c|c|c|}
\hline$\rho\left(\mathrm{kg} / \mathrm{m}^{3}\right)$ & $c(\mathrm{~m} / \mathrm{s})$ & $S$ & $\Gamma$ & $\mathrm{G}(\mathrm{GPa})$ \\
\hline 2750 & 5350 & 1.339 & 2.0 & 25 \\
\hline
\end{tabular}

$\Gamma$ : Gruneisen constant, $v\left(\frac{\partial p}{\partial v}\right)_{v}$.

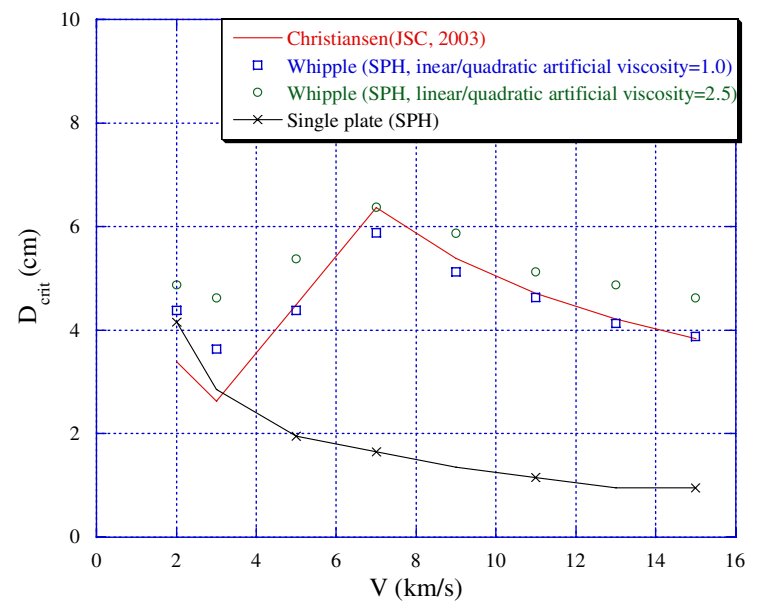

Fig. 3. Comparison of the critical diameter of a sphere as a function of impact velocity for fixed plate thickness. Other models are from the references.

for EOS are listed in Table 1, where $c$ is the speed of sound, and $G$ the shear modulus.

\section{Ballistic limit curves (BLCs)}

A series of simulations are conducted and the ballistic limit curves are obtained and shown in figure 3. The failure criterion was either "perforation" or "spall" whichever comes first. Just for comparison purpose, the ballistic limit curve for single aluminum plate was obtained and included in the figure. Absolutely, there is a gain for Whipple shield design compared with singe plate of equal total thickness. The generic empirical curve given by Christiansen is also compared together. In the current SPH simulations, we played with the artificial viscosities from 1.0 to 2.5 . We obtained slightly different results, indicating some affect of that parameter. In our code, a fairly well mach is obtained with the value of 1.0. However, this may not be the case for other group of SPH code. Furthermore other default constant setting also introduces some variances code by code. A special care is required before making some conclusions from this type of SPH simulations. It may rely on the user's experience and knowledge.

It is known that there are three regimes; ballistic regime for $\mathrm{V}<3 \mathrm{~km} / \mathrm{s}$, intermediate regime for $3<\mathrm{V}<7 \mathrm{~km} / \mathrm{s}$, and higher velocity regime for $\mathrm{V}>7 \mathrm{~km} / \mathrm{s}$. These are well simulated in this study. However it can be said that the slope for each regime is slightly different compared with the empirical curve. Especially for the high velocity regime the slope of predictions is flatter than the empirical data, and this is the case for most of the previous simulation 


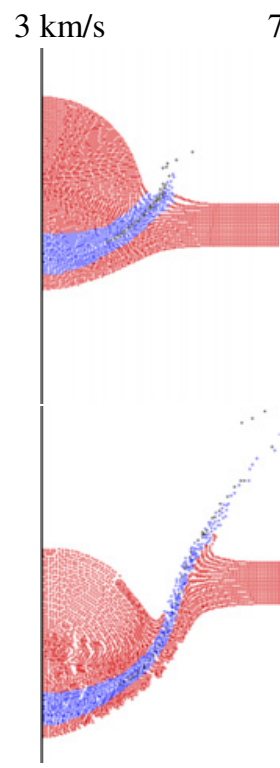

$\mathrm{T}=1,2.5$ us

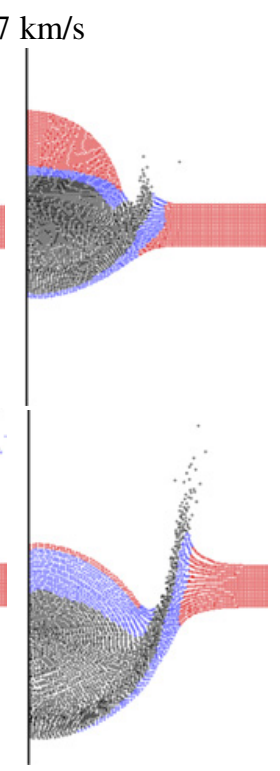

$\mathrm{T}=0.5,1 \mathrm{us}$

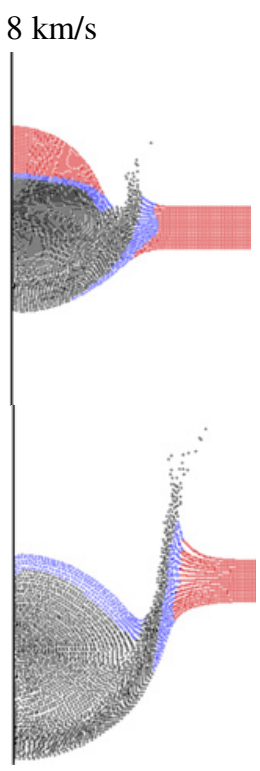

$\mathrm{T}=0.51 \mathrm{us}$

Fig. 4. Phases of a sphere and a plate during first bumper plate impact, read (solid state), blue (liquid state, Tmelt $=925 \mathrm{~K}$ ), black (vapor, Tvap $=2792 \mathrm{~K}$ ).

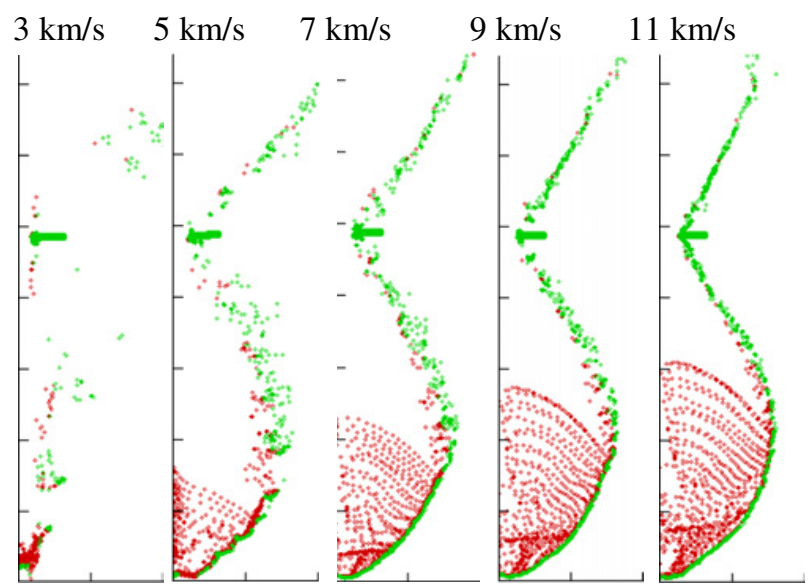

Fig. 5. The shape of debris just before backwall impact as a function of impact velocity.

studies. Even though the phase change is not taken into account either into the equation of state or energy equation as a discontinuous jump in energy level, a good result is obtained. Actually it seems that this is not the source of the error.

On the other hand we examined the phases of the material by calculating temperature from the energy. For T6-6061 aluminum, the melting temperature is $925 \mathrm{~K}$ and vaporization temperature is $2792 \mathrm{~K}$. The material phases are shown in the Fig. 4 as a function of impact velocity. For $\mathrm{V}=2,3 \mathrm{~km} / \mathrm{s}$, only the interface zone between the sphere and bumper plate becomes liquid (melting is observed), while the sphere starts to experience fragmentation. For high velocity $\mathrm{V}=7,8 \mathrm{~km} / \mathrm{s}$, the vapor zone as well as liquid zone is observed. From this velocity, it is known as a complete melting regime and consistent with the observation of Christiansen [6].

In Fig. 5, the debris cloud is compared as a function of impact velocity. This is the frame just before the impact onto the backwall plate. For $\mathrm{V}=3 \mathrm{~km} / \mathrm{s}$, the radial spreading is much less and is considered to be the ballistic regime. The affect of the bumper plate is restricted. For $\mathrm{V}>3 \mathrm{~km} / \mathrm{s}$ up to $7 \mathrm{~km} / \mathrm{s}$, a significant debris cloud is formed in radial extent due to fragmentation, resulting in pour penetration into the backwall plate (intermediate regime). For $\mathrm{V}>8 \mathrm{~km} / \mathrm{s}$, it seems that no further significant radial spreading is not observed, while the backward spreading on the backside of the sphere does not stop. This trend is responsible for the performance of the ballistic limit curve. More detail investigation is necessary.

\section{Conclusions}

A series of hypervelocity plate impact simulations was performed in axi-symmetric coordinate for certain geometry (the bumper thickness $1.6 \mathrm{~mm}$, spacing $120 \mathrm{~mm}$, and the backwall thickness $3.2 \mathrm{~mm}$ ). The impact velocity ranged from $2 \mathrm{~km} / \mathrm{s}$ to $14 \mathrm{~km} / \mathrm{s}$. The ballistic limit results calculated are compared with predictions from empirical models which are available in the literatures. The SPH predictions using in-house code are found to match fairly well with the empirical correlations using simple a linear shock equation of state and with no consideration of phase change. It explains that the equation of state is not the only error source, possibly other possibilities, such as different default setting in each code, some bugs in the computer code, different material properties, particle resolutions, and finally in the resolution of determining the failure (ballistic limit or spall limit.

Some activities are put to characterize the ballistic limit curve. Although these may not be enough, the capture of material phases and the debris cloud spreading as a function of impact velocity can provide a good insight on the hypervelocity thin plate impacts.

\section{Acknowledgements}

This work was supported by the Research fund of Survivability Technology Defense Research Center of Agency for Defenese and Develoment (UD090090GD).

\section{References}

1. Y.G. Angel, J.P. Smith, Int. J. of Impact Engng., 14, 25 (1993)

2. R. Kinslow, High-Velocity Impact Phenomena (Academic Press, New York, 1970)

3. B.G. Cour-Palas, J.L. Crews, Int. J. of Impact Engng., 10, 135 (1990)

4. A.J. Piekutowski, Int. J. Impact Engng. 14, 573 (1993)

5. E.L. Christiansen, Int. J. of Impact Engng., 14, 145 (1993) 
6. E.L. Christiansen, J.H. Kerr, Int. J. of Impact Engng., 26, 93 (2001)

7. D. Palmieri, M. Faraud, R. Destefanis, M. Marchetti, Int. J. Impact Engng. 26, 579 (2001)

8. W.W. Evans, R. Stallworth, Structures, Structural Dynamics, and Materials, 45 ${ }^{\text {th }}$ AIAA/ASME/ ASCE/AUS/ASC, AIAA (2004)

9. L.B. Lucy, The Astro. J. 82, 1013 (1977)

10. R.A. Gingold, J.J. Monaghan, Monthly Notices Royal Astr. Soc. 181, 375 (1977)
11. L.D. Libersky, A.G. Petschek, Advances in the FreeLagrange Method, (Lecture Notes in Physics, 395, Springer, 1990)

12. G.R. Johnson, E.H. Petersen, R.A. Stryk, Int. J. Impact Engng. 14, 385 (1993)

13. G.R. Johnson, R.A. Stryk, S.R. Beissel, Comput. Methods Appl. Mech. Engng. 139, 347 (1996)

14. C.J. Hayhurst, R. Clegg, Int. J. Impact Engng. 20, 337 (1997)

15. A.C. Mitchell, W.J. Nellis, J. Appl. Phys. 52 (1981) 